\title{
APLICACIÓN DE UNA PROPUESTA TEÓRICA Y METODOLÓGICA PARA LA PRODUCCIÓN DE DOCUMENTOS EN EL MODELO POR COMPETENCIAS: UN ESTUDIO CUASIEXPERIMENTAL
}

IMPLEMENTATION OF THEORETICAL AND METHODOLOGICAL PROPOSAL TO PRODUCE DOCUMENTS BY THE COMPENTENCY MODEL-QUASI-EXPERIMENTAL RESEARCH

Volumen 11, Número 1

pp. 1-31

Este número se publicó el 30 de abril de 2011

Ma. Isabel Araya Muñoz

Katia Varela Córdoba

La revista está indexada en los directorios:

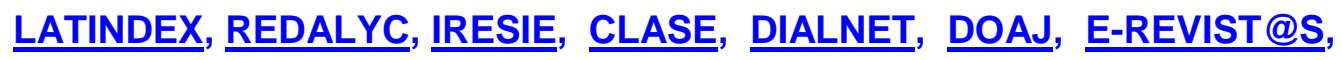

La revista está incluida en los sitios:

REDIE, RINACE, OEI, MAESTROTECA, PREAL, HUASCARAN, CLASCO 


\title{
APLICACIÓN DE UNA PROPUESTA TEÓRICA Y METODOLÓGICA PARA LA PRODUCCIÓN DE DOCUMENTOS EN EL MODELO POR COMPETENCIAS: UN ESTUDIO CUASIEXPERIMENTAL

\author{
IMPLEMENTATION OF THEORETICAL AND METHODOLOGICAL PROPOSAL TO PRODUCE
} DOCUMENTS BY THE COMPENTENCY MODEL-QUASI-EXPERIMENTAL RESEARCH
}

\author{
Ma. Isabel Araya Muñoz ${ }^{1}$ \\ Katia Varela Córdoba ${ }^{2}$
}

\begin{abstract}
Resumen: Este artículo muestra los resultados de un estudio cuasiexperimental, definido como Diseño de Grupo Control sin Tratamiento. Se elaboró y se implementó una propuesta teórica y metodológica para el proceso de enseñanza y aprendizaje para la producción de documentos en las oficinas, en el enfoque por competencias, en un curso de las carreras Administración de Oficinas y Educación Comercial de la Escuela de Secretariado Profesional de la Universidad Nacional de Costa Rica, primer ciclo 2009. Tres grupos participaron: uno experimental con tratamiento y dos grupos control con la metodología tradicional. Los resultados del postest mostraron que el experimental superó el promedio de rendimiento en 6,5 puntos al grupo control 2 y en 1,13 puntos al grupo control 1, línea que se marca en la comparación del rendimiento por áreas cognoscitivas. Para complementar los resultados de los tests se exploraron también factores del proceso de enseñanza y de aprendizaje aplicado en cada grupo. Además, mediante técnicas cualitativas se identificó la incidencia en el rendimiento de las estrategias de aprendizaje, el interés de los estudiantes y las estudiantes, su experiencia laboral, la formación dual y la carrera así como el papel de la persona docente. Se concluye que el modelo de formación por competencias podría mejorar el rendimiento académico en la producción de documentos cuando un espacio pedagógico es abordado desde la integración de saberes a partir de actividades didácticas que fomenten el diálogo, la indagación, la aplicación teórica a la resolución de problemas, el aprendizaje experiencial en el aula y en entornos lo más relacionados con la futura práctica profesional.
\end{abstract}

Palabras clave: EDUCACIÓN POR COMPETENCIAS, ESTUDIO CUASI-EXPERIMENTAL, PRODUCCIÓN DE DOCUMENTOS, OFICINA, SECRETARIADO, ADMINISTRACIÓN DE OFICINAS

\begin{abstract}
This article shows the results of a quasi-experimental study defined as Control Group Design without Treatment. A theoretical and methodological proposal was carried out and implemented for the teaching and learning processes for document production in the offices, focusing in the competences, the Office Management and Commercial Education courses in the careers taught at the Escuela de Secretariado Profesional at the Universidad Nacional de Costa Rica, first cycle 2009. Three groups participated: one experimental with treatment and two control groups with traditional methodology. The results of the post-test show that the experimental group went beyond the average performance by 6.5 points compared to control group 2 and by 1.13 points compared to control group 1 , difference which becomes more obvious when comparing the performance in cognitive areas. The process of teaching and learning applied in each group were also explored and through quality techniques the incidence in the performance of learning strategies, interest of the students, their work experience, the dual education and the career as well as the role of the educator were identified to complement the results of the tests factors. It was concluded that the education through competences could improve the academic performance in producing documents when a pedagogical space deals with the integration of knowledge based on the didactic activities that promote dialogue, research, theory application to problem solving, practical learning in the classroom and in environments related to future professional practice.
\end{abstract}

Key words: COMPETENCY-BASED EDUCATION, QUASI-EXPERIMENTAL RESEARCH, DOCUMENTS PRODUCTION, SECRETARIAL WORK, OFFICE

\footnotetext{
${ }^{1}$ Licenciada en Educación Comercial. Máster en Educación. Académica: Universidad Nacional de Costa Rica, Profesora I, Investigadora: Escuela de Secretariado Profesional. Egresada Magíster en Planificación Curricular, Universidad de Costa Rica. Docente del Ministerio de Educación Pública de Costa Rica. Dirección electrónica: isaaraya@yahoo.com

2 Licenciada en Pedagogía, Académica: Universidad Nacional de Costa Rica, Profesora I, Investigadora: Escuela de Secretariado Profesional. Educadora del Ministerio de Educación Pública de Costa Rica. Dirección electrónica: katiavarela1@gmail.com
}

Artículo recibido: 27 de enero, 2011

Aprobado: 28 de marzo. 2011 


\section{Introducción}

En los últimos años, los países desarrollados han incursionado en un cambio hacia la globalización y la transnacionalización de la economía, por lo que cada vez son más los requisitos mundiales para la productividad y la calidad. Aunado a esto, se deben considerar los cambios sustanciales en las nuevas tecnologías que se incorporan a los procesos de producción y de negociación. El cambio no solo es llamado a las políticas y a los procedimientos, sino también a las nuevas capacidades humanas para que respondan a las exigencias de un desempeño eficiente.

La producción de documentos que se contempla en este estudio se refiere a los conocimientos teóricos y prácticos para la elaboración de diferentes tipos documentales en el curso de Técnicas Mecanográficas por Computadora III, del segundo nivel de la carrera de Administración de Oficinas y Educación Comercial de la Escuela de Secretariado Profesional de la Universidad Nacional de Costa Rica, la cual se realiza de acuerdo con la propuesta de textos educativos, con un enfoque de aprendizaje de contenidos y el abordaje de la temática alcanza la experticia de los docentes. En general, la planta docente realiza una adaptación de la metodología aplicada a la enseñanza de la mecanografía, a partir de adaptaciones de conceptos y formatos de otros contextos, sin una reflexión profunda sobre las necesidades propias.

La transferencia de la mecanografía a la producción de documentos en procesadores de textos involucra no solo un cambio de los procedimientos por seguir en términos de la competencia que se desea desarrollar, sino el papel del docente y los materiales o contenidos curriculares, así como la adecuación a los estándares de calidad nacional e internacional.

Esa transferencia de los procedimientos metodológicos en la producción documental se plantea también en la necesidad de propiciar la incorporación de nuevos enfoques educativos a las aulas universitarias con fines de promoción y desarrollo de particularidades en una interacción de aprendizaje, desarrollo de destrezas, habilidades, conocimientos y valores integrados con la cultura laboral. Por lo anterior, este estudio se basa en el argumento de que la producción de documentos en el modelo por competencias favorece el rendimiento académico.

Por esa razón, es conveniente realizar estudios sobre el desarrollo de la producción documental, debido a los nuevos retos de la apertura comercial, los avances en las Tecnologías de la Comunicación y la Información (TIC) y su aplicabilidad en los procesos organizacionales que exigen mejor desempeño en la elaboración de documentos. 
El propósito es analizar el rendimiento al implementar una propuesta teórica y metodológica para el proceso de enseñanza y de aprendizaje para la producción de documentos en las oficinas con un nuevo abordaje pedagógico. En cuanto a su estructura, el estudio se planteó en dos etapas: en la primera se elaboró una propuesta teórica metodológica para la producción de documentos en el modelo de enseñanza y de aprendizaje por competencias, en el ámbito del curso en estudio y en la segunda se trabajó en la aplicación de la propuesta a un grupo (experimental) y dos grupos (control) trabajaron con la metodología tradicional.

\section{REFERENTE TEÓRICO}

\subsection{La producción documental}

Los documentos contienen información y evidencia de las funciones, normas, decisiones, procedimientos, operaciones y otras actividades de toda organización, por ello, se convierten en medios para comprobar hechos.

La cualidad de comprobación que tienen los documentos se da a partir de su legalidad y visado, características que las dan las condiciones de acreditación de parte del ente que las emite, lo cual se confirma por medio de la fecha, la firma y el sello en su presentación original. El Código Procesal Civil (1989) establece que los documentos son medios probatorios en los procesos judiciales, y los define como todos los objetos muebles que tengan carácter representativo o declarativo.

Asimismo, en el ámbito costarricense, la Junta Administrativa del Archivo Nacional establece que un documento debe ser confiable y auténtico; se considera confiable cuando es emitido por la entidad correspondiente y con la debida autorización, y auténtico, si es original, real o fidedigno y está vigente (Gaceta No. 61,83. 2008, p.14, p.71). Esas dos cualidades dependen del contenido, de la estructura y del contexto en el que se producen, desde el punto de vista legal y administrativo.

Entonces, el valor de un documento se demuestra en su calidad de válido o confiable por el ente autorizado para producirlo y en su autenticidad, esto significa que tiene valor jurídico o administrativo, según la naturaleza por la que fue creado, entre esos valores se definen algunos como: financiero, contable, informativo, histórico, legal.

La calidad de válido y auténtico de un documento, la Ley 7202, Art. 33, la delega a órganos colegiados para que definan su vigencia según la naturaleza propia de cada organización para las diferentes fases de vida de los documentos. En las diferentes fases de la 
vida de los documentos, la vigencia administrativa, legal o histórica la propone el Comité Institucional de Selección y Eliminación y posterior refrendo de la Comisión de Selección y Eliminación de Documentos. Esta Comisión también estima el valor científico y cultural de los documentos.

De esta manera, los documentos se convierten en el patrimonio informativo de una empresa o institución, de un pueblo, de una nación y de la sociedad, ya que contienen datos e información que se constituyen en el medio para comprobar hechos pasados que permitirán analizar el proceder presente y futuro de las personas en los diferentes ámbitos sociales.

En las oficinas se elaboran documentos que contienen datos e información que respaldan las diferentes acciones y transacciones que se realizan allí, por lo tanto, dos de las significaciones del concepto producción que se relacionan con ese ámbito, y que el DRAE (2001) propone, son elaborar cosas útiles y entender ideas por medio de la palabra.

La producción de documentos, Cortés (1984) la relaciona con el proceso de digitar documentos, actividad que se realiza en un tiempo razonable y de acuerdo con las normas establecidas.

Esta actividad es una de las labores más cotidianas en una oficina y corresponde a la integración de los conocimientos intelectuales y técnicos sobre contenido y formato de los diferentes tipos documentales, habilidades y destrezas técnicas como dominio completo de la herramienta de procesamiento de datos o procesador de textos y equipo periférico, así como aspectos normativos o legales, los procedimientos técnicos y administrativos del quehacer organizacional; es decir, para producir una carta es importante que la persona conciba la necesidad de confeccionar este tipo documental, luego deberá reunir la información pertinente, técnica, normativa y procedimientos administrativos. Luego procede a redactarla, lo que involucra no solo habilidad para componer sino también conocimiento de las relaciones con otros procesos administrativos, aplicarle las características de formato, imprimirla, recoger la firma, sellarla; o en su defecto, si se utiliza la firma digital realizar la encriptación correspondiente. Una vez finalizado ese proceso de trámite pasa a ser conservada en el lugar adecuado para su consulta durante, su vigencia en la etapa de gestión.

Todas las empresas públicas o privadas producen documentos con información que les permite ejecutar las acciones propias de la actividad a la cual se dedican, ya sean instituciones de servicio como: salud, financieras, educativas, comerciales, turísticas, agropecuarias y otras; por lo tanto, de acuerdo con Hernández, Hernández, y Segura (2005) y la normativa vigente, en torno a la elaboración de los diferentes tipos documentales, es importante considerar sus 
características internas y externas, cada documento, identifica su origen y razón por la que fue creado, así como la necesidad de su producción y su utilización específica depende de sus características especiales y de la normativa empresarial o institucional.

La elaboración de documentos en las oficinas ha evolucionado tanto como surgen nuevos medios tecnológicos y cambios en las normas y en los procedimientos, por ello, se requieren nuevos conocimientos, destrezas y habilidades; por lo tanto, conlleva la integración de aspectos teóricos y prácticos sobre formato, normativa, herramientas tecnológicas, normas de salud y seguridad, estándares de calidad, gramaticales y procedimientos administrativos.

\subsection{Las estrategias de aprendizaje}

Las estrategias de aprendizaje orientan hacia la construcción del conocimiento de la persona estudiante, Monereo, Castelló, Palma y Pérez (1999), las definen como un proceso de toma de decisiones consciente e inconscientemente, en los cuales se eligen y recuperan, de manera coordinada, los conocimientos que se necesitan para cumplir los objetivos o metas. En ese proceso intervienen cuatro preguntas claves: ¿qué? la actividad de aprendizaje, ¿cómo? las técnicas y estrategias que utilizará, ¿para qué? aprender contenidos y ¿por qué? la meta o la finalidad.

Este autor también reflexiona sobre las estrategias de aprendizaje como procesos dirigidos a un objetivo de aprendizaje y desde la docencia se orientan hacia la persona que aprende, por lo tanto, se debe tomar en cuenta qué saben, qué desconocen, qué quieren aprender y cuánto aprenden y buscar mejorar el conocimiento declarativo, procedimental y condicional o estratégico.

Además de esa relación entre las estrategias, la cognición y el fin u objetivo, Pozo (1990), toma en cuenta en el aprender los procesos básicos (información presentada y una determinada capacidad de memoria a largo plazo), conocimientos relativos a diversas materias, estrategias de aprendizaje como secuencia planificada de actividades que realiza el sujeto con el fin de aprender, metaconocimiento o conocimiento sobre sus propios procesos psicológicos, distingue estrategias y habilidades de estrategias de apoyo.

En tal sentido y para el logro de los diferentes tipos de conocimiento, son necesarias las estrategias de aprendizaje como intenciones desde la docencia y en función de la persona e involucran métodos y técnicas, estrategias, habilidades y procedimientos. 
Considerando que, en el ámbito educativo, el nivel de aprendizaje de una persona está relacionado con la metodología utilizada, hay una estrecha relación en la construcción del aprendizaje y los instrumentos conceptuales y motivacionales que se empleen en un proceso de enseñanza, éstos se constituyen en un conjunto de pasos intencionales para que logre significatividad en los contenidos adquiridos y, por ende, esto influye en el rendimiento académico.

Las estrategias de aprendizaje, por lo tanto, se conciben como un proceso metodológico innovador, centrado en el diálogo, el trabajo en equipo, individual y colaborativo, autónomo de parte de la persona estudiante, involucrado y protagonista.

En ese proceso, el papel del docente y de la docente es de orientación para generar espacios para la discusión entre la población estudiantil, con la persona docente y con personas especialistas externos. Esas características de un proceso educativo, Montero, Villalobos y Valverde (2007) las consideran como diferentes a una enseñanza tradicional y favorecen el rendimiento académico.

\subsection{El rendimiento académico}

Para analizar el rendimiento se tomaron en cuenta algunas conceptualizaciones que se le han dado a este elemento en el proceso de enseñanza y de aprendizaje, se estudiaron las propuestas de trabajos de investigación en el ámbito latinoamericano y europeo, tales como las de Badilla (s.f.); Demagistri (2009); Zapata, De los Reyes, Lewis y Barceló (2009); Montero, Villalobos y Valverde (2007); Molero Lopez-Barajas (2007); García, Alvarado y Jiménez (2000), quienes en sus estudios abordaron el rendimiento académico.

A partir de las propuestas de estos autores, el rendimiento apunta al producto de un proceso y en el ámbito académico y la cantidad y calidad de conocimiento adquirido por una persona, es decir, al nivel de aprendizaje logrado y que está influido por diversos comportamientos propios de la persona que estudia, internos del proceso de enseñanza y de aprendizaje en el aula, así como de su contexto, puede estar respresentado en una nota al final de un proceso educativo, considerando que esa medición se ha dado a partir de una serie de actividades evaluativas en el transcurso.

\subsection{Las competencias en educación}

Las competencias en educación han sido tema de estudio en las últimas décadas con miras a la búsqueda de la mejora educativa, especialmente a relacionar la teoría con la práctica. 
Esa relación, conocimiento-experiencia, ha llevado a orientar la enseñanza únicamente hacia la tarea, al saber hacer, dejando del lado el desarrollo integral de la persona.

Por esto, es común encontrar relaciones muy específicas con el concepto de competencias, a modo de ejemplo, las siguientes: rendimiento en una situación de trabajo, cualificación, capacidades para la tarea, conductas que posee la persona y que asegura el éxito en la función, poner a prueba en situaciones de trabajo, capacidad para hacer tareas y situaciones de trabajo, capacidad demostrada en una organización, capacidad productiva del individuo, posibilidad de desempeño y competitividad,

Ese abordaje de las competencias procura acercar la academia a la práctica en un puesto de trabajo para hacer más eficiente la formación profesional; no obstante, los proyectos en esta modalidad discurren en saber hacer o aplicar lo aprendido, pero su propósito va más allá y se pretende que las competencias actúen como guías para la confección y desarrollo de los currículos y de las políticas educativas.

En ese sentido, la reflexión más importante debe centrarse en los fines educativos y que se deben establecer en un proyecto educativo que dimensione ¿qué aprender? ¿cómo aprenderlo? y ¿para qué aprenderlo?, cuyas respuestas dependerán de la visión de la educación. Kemmis (1998) indica que desde la aparición de la educación de masas, la planificación curricular ha tratado de transformar la sociedad mediante cambios en los currículo y en la escolarización, por ello, el currículum no debe considerarse solo como la organización de lo que debe ser enseñado y aprendido.

Desde la educación, las competencias son el medio para el saber, el saber hacer, el ser y el convivir que fortalezcan a la persona en la toma de decisiones acertadas, con expresión de libertad, de coexistir con justicia y respeto y emprender proyectos con éxito adaptando lo aprendido a las diversas situaciones de la realidad profesional futura.

La educación, así orientada, permite innovar, es decir, aprender de la realidad para transformarla y a partir de las ideas de Villarini (1996), el Proyecto Tuning en el marco de la Declaración de Bolonia (Proyecto Tuning-2005), Arias (2005), Gimeno, Pérez, Martínez, Torres, Angulo, y Álvarez (2009), Villa y Poblete (2004), Tobón (2005), Perrenoud (1999), el modelo pedagógico de la Universidad Nacional (Costa Rica, Universidad Nacional. sf) y la perspectiva de la educación centrada en la persona estudiante es como se elaboró la propuesta que se detalla en el siguiente apartado. 


\section{PROPUESTA ESTRATEGIAS METODOLÓGICAS PARA LA PRODUCCIÓN DE DOCUMENTOS EN EL MODELO POR COMPETENCIAS}

\subsection{Fundamentos teóricos de la propuesta}

La producción de documentos, en el modelo por competencias, se visionó considerando los fines de la educación costarricense, los principios de la educación universitaria costarricense y específicamente los de la Universidad Nacional y los objetivos estratégicos de la Escuela de Secretariado Profesional.

En el nivel universitario, y en correspondencia con los fines de la educación costarricense, la formación se enfoca desde tres perspectivas de desarrollo: social, democrático y en ciencia y tecnología. Esas orientaciones se establecen en la misión de la Educación Superior Costarricense, cuyo ideal de formación de ser humano, según Consejo Nacional de Rectores (2005), se fundamenta en un marco filosófico sobre la pregunta por el ser, el sentido del cambio y el concepto de sujeto. A partir de esa premisa, la labor universitaria provee principios y valores comunes que guían las acciones estratégicas para la búsqueda de la excelencia, la transparencia, la pertinencia, la formación humanística, la formación científica, la equidad, la innovación y la creatividad.

En congruencia con los principios del Plan Nacional de Educación Superior, la Universidad Nacional, como parte de ese sistema de educación universitaria estatal, está llamada a romper con los enfoques mercantilistas y se postula hacia la innovación, la solidaridad y el conocimiento científico en la búsqueda de la excelencia, sin perder de vista la formación de los estudiantes y las estudiantes con fundamento en una filosofía humanista (Costa Rica, Universidad Nacional, 2004).

Por ello, el marco filosófico orientador del desarrollo de su quehacer se contempla en la misión, visión, principios y valores que, en concordancia con la planeación estratégica de la educación universitaria a nivel nacional, guían su labor (Costa Rica, Universidad Nacional. 2004). Ese trabajo lo realiza a través de las unidades académicas y administrativas, según la estructura orgánica de la Universidad Nacional, cuyas funciones se planean estratégicamente considerando las políticas y fines educativos institucionales.

Los profesionales graduados y graduadas de la Universidad Nacional se deben caracterizar por ser personas formadas integralmente, capaces de aportar a los procesos productivos en los cuales se insertarán en su vida laboral, por lo que deben dar su aporte intelectual a la solución de los problemas de su entorno, así como tener la capacidad socio- 
afectiva que les permita una buena calidad de vida con los demás seres que les rodean, de esa manera se fortalecerá la convivencia en paz y en solidaridad.

Por consiguiente, la Universidad Nacional y la Escuela de Secretariado Profesional proponen en su misión un concepto de educación que rige la actividad académica como el desarrollo de facultades intelectuales y morales de la persona para que pueda desenvolverse en su vida laboral con éxito y así aporte al desarrollo económico del país con competencia, no con la competitividad que busca el capitalismo, sino con bases de productividad con conciencia de los derechos y valores humanos para la convivencia en sociedad con los más altos principios, promoviendo una educación que propicie la integración del saber, del hacer, del ser y el convivir.

Considerando el contexto donde se enmarca el estudio sobre rendimiento en la producción de documentos, es necesaria la integración curricular que facilite el contacto de los estudiantes y de las estudiantes con la realidad productiva, ya que las competencias parten de ese contexto para que, tal y como lo afirma Araya (2008), en esa relación se lleve a cabo un aprendizaje realidad. Desde esa perspectiva se plantea una concepción de hombre y de mujer que aporte a la convivencia en sociedad.

En ese entorno, el enfoque educativo que se plantea se centra en la persona estudiante y parte desde perspectivas humanistas y tecnológicas, con miras a fortalecer la formación desde un sistema de gestión por competencias con miras a la formación profesional con alta capacidad productiva y comprometido con los valores para la convivencia y desarrollo de sus potencialidades.

Alonso, Gallego y Honey (1994) mencionan algunos principios que Carl Rogers describe sobre este enfoque curricular como experiencia integrada o de realización personal partiendo de que la persona puede aprender cuando tiene interés y así logra un aprendizaje significativo. En ese proceso influye la percepción de sí mismo, interviene el ego, los sentimientos, la participación, la práctica y la socialización.

Este punto de vista de la educación lo fundamenta Dewey (1960), al indicar que el principio para que la educación alcance los fines respecto al individuo y a la sociedad tiene que basarse en la experiencia, la cual es vital para su desarrollo académico y profesional.

La integración de esa relación teoría-práctica se plantea a partir del aprendizaje por competencias tratando de relacionar a la persona estudiante con nuevas estrategias de aprendizaje por medio de las cuales pueda aplicar conocimientos, seleccionarlos y disponerlos en las condiciones que influyan en su experiencia presente, por lo que la institución educativa 
debe brindar una posibilidad de experiencia creciente o progresiva. No se trata de orientar la educación únicamente hacia el quehacer organizacional sino, más bien, hacia la integración de la utilidad, el conocimiento teórico y técnico, las aptitudes y las condiciones, medios, recursos y estrategias.

La aplicación metodológica del aprendizaje por competencias se sustenta en la estructura propuesta por el Proyecto Tuning en el marco de la Declaración de Bolonia (Proyecto Tuning América Latina, 2005). Este método trata de impulsar la integración de la diversidad, la libertad y la autonomía en los proyectos educativos basados en la formación por competencias, toma en cuenta dentro de su estrategia cuatro componentes: las competencias genéricas y específicas, los enfoques de aprendizaje, didácticos y de evaluación, la función de la promoción de la calidad en los procesos educativos y la equiparación de créditos.

La producción de documentos en el modelo por competencias se aborda considerando los tres primeros componentes de la metodología Tuning, ya que el interés específico se centra en los procesos aplicables a la producción de documentos en las oficinas en el contexto de las carreras en estudio.

¿Por qué Tuning? Las universidades costarricenses han aprovechado la plataforma de cooperación regional para enriquecer los planes de estudio, tal y como lo mencionan Beneitone, Esquetini, González, Maletá, Siufi, y Wagenaar (2007), el Consejo Centroamericano de Educción Superior (CSUCA) asume este proyecto como un reto para las universidades, ya que promueve el proceso de reforma de toda la Educación Superior en la región y garantiza la acreditación y el mejoramiento permanente.

Además, se consideran las propuestas de Delors, Mufti, Amagi, Carneiro, Chung, Geremek, Gorham, Kornhauser, Manley, Padrón, Savané, Singh, Stevenhager, Won Suhr, y Nanzhao (1996), ideas de Villarini (1996), el Proyecto Tuning América Latina (Proyecto Tuning2005), Arias (2005), Gimeno, Pérez, Martínez, Torres, Angulo, y Álvarez (2009), Villa y Poblete (2004), Tobón (2005), Perrenoud (1999) y otros autores que visualizan la formación por competencias desde un enfoque de aprender integral, así como los lineamientos del modelo pedagógico de la Universidad.

En ese sentido, la propuesta teórica metodológica para el desarrollo de las competencias para producir documentos en el ámbito del curso en estudio, considera los siguientes elementos: 
- Enfoque educativo centrado en la persona estudiante, con una perspectiva humanista, orientada hacia la integralidad de saber, saber hacer, ser y convivir, conducente hacia un aprendizaje realidad.

- Competencias genéricas como ejes transversales que fortalecen el currículo en cuanto actitudes y valores; competencias específicas que parten de un propósito general o competencia global, la cual genera tres problemas de la práctica profesional, de allí se derivan las competencias específicas, los elementos de competencia y sus ejes temáticos.

- Metodología de enseñanza y de aprendizaje dinámica e interactiva, la persona docente guía el proceso pedagógico, la persona estudiante activa y responsable de su propio aprendizaje, aplicación de situaciones de aprendizaje que acerquen al estudio de la realidad por medio de prácticas del contexto y en entornos lo más relacionados con la futura práctica profesional; la interdisciplinariedad como eje fundamental en la integración de saberes y la evaluación como medio para aprender.

Considerando esos elementos, la competencia global que enmarca el curso en investigación integra los conocimientos intelectuales, procedimientos técnicos, administrativos y legales; destrezas y habilidades pertinentes, actitudes necesarias para el logro de resultados con eficiencia y calidad para la producción de informes, actas y documentos conexos, y currícula vitae y se define como:

Producir informes, actas y documentos conexos y currícula vitae, que se requieren en las oficinas, con los conocimientos intelectuales, procedimientos técnicos, administrativos y legales propios de cada tipo documental, con la aplicación de las destrezas para el uso de las herramientas tecnológicas requeridas y las habilidades pertinentes, actitudes necesarias para el logro de resultados con eficiencia, calidad, autonomía, que le permitan a la persona estudiante adaptar a situaciones nuevas.

\subsection{Competencias genéricas}

En el caso de las carreras en estudio y debido a la carencia de la definición de las competencias genéricas, referidas a los comportamientos asociados con el desempeño, común a diversas ocupaciones y ramas de actividad productiva, se procedió a realizar un proceso de selección de las competencias genéricas aplicables a la universidad y, por ende, a las carreras en estudio. 
Se consideraron las 27 competencias genéricas seleccionadas por el Proyecto Tuning América Latina, que según Beneitone et. al. (2007) fueron escogidas de un listado de 85, que académicos y académicas, población estudiantil, personas graduadas, así como contratantes, entre 19 países y 190 universidades latinoamericanas de 12 áreas temáticas o carreras universitarias comunes, consideraron con mayor grado de importancia y de realización de cada una.

Partiendo de esas 27 competencias genéricas, debidamente concordadas con los principios del Modelo Pedagógico de la Universidad Nacional, se sometieron a consulta sobre el grado de importancia, a académicos de esta universidad, involucrados en proyectos relacionados con el tema de competencias, así como con procesos de acreditación de carreras. De esta forma, se seleccionaron cuatro competencias genéricas que se aplicaron como marco de referencia para elaborar la propuesta teórica metodológica para la producción de documentos, las cuales son las siguientes:

\begin{tabular}{|l|}
\hline \multicolumn{1}{|c|}{ Tabla No. 1. } \\
Competencias Genéricas para la Propuesta \\
\hline Capacidad de trabajo en equipo \\
\hline Compromiso con la calidad \\
\hline $\begin{array}{l}\text { Capacidad para identificar, plantear y resolver } \\
\text { problemas }\end{array}$ \\
\hline Compromiso ético \\
\hline Fuente: Elaboración de las autoras \\
\hline
\end{tabular}

\subsection{Competencias específicas}

Las competencias específicas que identifican los comportamientos asociados a conocimientos técnicos de una tarea, en este caso la producción de documentos, corresponden a la integración de los conocimientos intelectuales y técnicos sobre contenido y formato de los diferentes tipos documentales, destrezas técnicas como dominio completo de la herramienta de procesamiento de datos o procesador de textos y equipo periférico, así como los procedimientos técnicos y administrativos del quehacer organizacional.

A partir del análisis de la integración del saber, saber hacer, saber ser y convivir en la práctica profesional en el ámbito de las oficinas, se plantearon tres problemas fundamentales: 
1. ¿Cuáles son los requerimientos conceptuales y los procedimientos técnicos, administrativos y legales sobre contenido y formato para la elaboración de actas y los documentos conexos que se requieren en las oficinas?

2. ¿Cuáles son los requerimientos conceptuales y los procedimientos técnicos, administrativos y legales sobre contenido y formato para la elaboración de los diferentes tipos de informes que se utilizan en las oficinas?

3. ¿Cuáles son los requerimientos conceptuales y los procedimientos técnicos, administrativos y legales sobre contenido y formato para la elaboración de currícula vitae que se requieren en las diferentes oficinas?

A partir de la búsqueda de respuestas a esos ejes problemáticos en torno a la competencia global se definieron las competencias específicas, de las cuales se derivan los elementos de competencias y de éstos los ejes temáticos.

\subsection{Orientación metodológica de la propuesta}

La orientación metodológica de la enseñanza por competencias aplicable a la producción de documentos en las oficinas en el contexto de las carreras en estudio, parte en primera instancia de una competencia global, de esta a unos problemas de la práctica profesional, de los cuales resultan las competencias específicas, los elementos de competencia y su desarrollo temático. Por esa razón, se plantea como un proceso formativo, dinámico, participativo e interactivo, basado en el aprendizaje cooperativo y autónomo, en actividades que integren la consulta permanente de los procedimientos y normativa propia de las oficinas mediante pasantías cortas y trabajos de observación y consulta; así como del apoyo de aula virtual para lograr una comunicación permanente entre las personas participantes, trabajos de consulta a profesionales de las diferentes disciplinas relacionadas. En el aula, por lo tanto, las estrategias de aprendizaje parten del ejemplo real.

A la persona docente le corresponde asumir un papel de generador de espacios didácticos propicios para el desarrollo de aprendizaje significativo y lo que esto conlleva, la recopilación de evidencias en el proceso con fines de mejora, acudir a la consulta y realimentación de las diferentes áreas disciplinarias de la carrera, la cual a pesar de que en esta propuesta no cambia la malla curricular, desde el curso o espacio pedagógico donde se enmarca la producción documental, este proceso debe ser abordado con visión integrada de los 
saberes, lo que involucra la interacción permanente entre colegas de las áreas de conocimiento de las carreras.

Además, la propuesta se desarrolla con apoyo tecnológico, por medio de la plataforma del Aula Virtual con las herramientas: el portafolio reflexivo cooperativo, que consiste en la narración semanal de las experiencias más significativas en su proceso de aprendizaje con respecto a las temáticas tratadas; también el foro de discusión con personas expertas gestionado por los estudiantes y las estudiantes y el glosario pictórico en el cual comparten diferentes significados, su aplicación, su comentario y su ilustración.

La idea fundamental de la propuesta en el modelo por competencias es plantear un cambio de la pedagogía tradicional, tan calada en las aulas universitarias. En la Tabla No. 2 se contrastan las dos perspectivas formativas.

\begin{tabular}{|l|l|l|}
\hline \multicolumn{1}{|c|}{ Tabla No. 2} \\
Contrastación del Modelo Pedagógico Tradicional y el Modelo por Competencias
\end{tabular}




\begin{tabular}{|c|c|}
\hline & $\begin{array}{l}\text { - La persona estudiante posee competencia cognitiva para } \\
\text { aprender y solucionar problemas, es responsable de su } \\
\text { propio aprendizaje y aprende a aprender y a pensar. } \\
\text { - La educación se orienta al aprendizaje significativo. } \\
\text { - La persona docente planea y organiza experiencias para } \\
\text { el aprendizaje, no se centra en la enseñanza. Es } \\
\text { generador de procesos de construcción individual y social, } \\
\text { propone preguntas, casos, problemas y prácticas de } \\
\text { contexto. }\end{array}$ \\
\hline $\begin{array}{l}\text { Conocimientos } \\
\text { basados en libros de } \\
\text { texto }\end{array}$ & $\begin{array}{l}\text { Construcción de conocimiento asociado a la realidad } \\
\text { productiva y a la experiencia de los participantes en torno a } \\
\text { los problemas de su realidad y la relación con su futuro } \\
\text { profesional. }\end{array}$ \\
\hline $\begin{array}{l}\text { Evaluación directiva y } \\
\text { con fines de control }\end{array}$ & $\begin{array}{l}\text { Promueve procesos de reflexión sobre lo aprendido, mediante } \\
\text { la integración de la evaluación directiva, autoevaluación y } \\
\text { coevaluación. La evaluación es una oportunidad de mejora. }\end{array}$ \\
\hline
\end{tabular}

Considerando lo anterior, el curso se diseñó incluyendo como metodología básica: a) descripción integrada de las competencias que se esperaba desarrollar en el espacio pedagógico, aclarando el vínculo entre ambos tipos de competencias, genéricas y específicas, especialmente, para centrar el enfoque hacia la persona estudiante, b) demostrar la transversalidad de las competencias genéricas, c) el objeto de las competencias específicas y sus contenidos temáticos, d) las estrategias de aprendizaje orientadas hacia la diversidad con sus características, intereses y necesidades diferentes que propicien aprendizajes autónomos, e) los indicadores de logro o estrategia evaluativa tanto de las competencias específicas como de las genéricas, aplicables en el inicio, en el proceso y al final, y f) las fuentes de consulta obligatorias y recomendadas.

En ese sentido, la propuesta de formación basada en competencias para la producción de documentos comprende los conocimientos intelectuales, procedimientos técnicos, administrativos y legales sobre las características internas y externas de los diferentes tipos documentales, así como las destrezas para el uso de las herramientas tecnológicas requeridas y las habilidades pertinentes para la aplicación de los procedimientos adecuados, actitudes y valores necesarios para el logro de resultados con eficiencia, calidad, autonomía y sentido de la realidad.

\section{METODOLOGÍA}

El diseño de investigación aplicado corresponde a un estudio cuasiexperimental, pretestpostest, definido como Diseño de Grupo Control sin Tratamiento, clasificado así entre las Volumen 11, Número 1, Año 2011, ISSN 1409-4703 
variantes de los Diseños de Grupo Control no Equivalente por Cook y Campbel (1979), citados por Kerlinger y Lee (2002). A partir de la participación de tres grupos, a uno de ellos se le aplicó tratamiento y dos fungieron como grupos control. El pretest se aplicó al inicio del curso y el postest al finalizar.

La población total corresponde a los tres grupos del curso Técnicas Mecanográficas por Computadora III, código SPJ212, del segundo nivel de las carreras de Administración de Oficinas y Educación Comercial de la Escuela de Secretariado Profesional, segundo ciclo 2009, en total 58 estudiantes. La muestra de investigación fue el total que realizó el pretest y postest, en total 36 personas, quienes se consideraron equiparados en cuanto al nivel académico, ya que habían cumplido con los requisitos anteriores de la carrera.

La muestra se define como no aleatoria, por lo que la selección de las personas participantes depende del objeto de la investigación. Los estudiantes y las estudiantes del grupo experimental y de los grupos control se consideran apareados en términos del nivel de formación académica.

\subsection{Test o prueba escrita}

El pretest y postest consistió en una prueba escrita, originalmente conformada por 35 ítemes sobre los diferentes saberes para medir el rendimiento en la producción de documentos, considerando las áreas de conocimiento: saber (conocimiento fácticos), hacer (saber hacer, procedimental) ser y convivir (actitudes, valores, disposición, competencias genéricas). Esos factores contemplados en el desarrollo de la competencia general, se definen como: integración de conocimientos intelectuales, procedimientos técnicos, administrativos y legales; destrezas y habilidades pertinentes, actitudes necesarias para el logro de resultados con eficiencia y calidad para la producción de informes, actas y documentos conexos y currícula vitae.

La validez del instrumento se realizó mediante validación de contenido, que Kerlinger y Lee (2002) definen como la representatividad del contenido de un universo de contenido, es decir, los reactivos del instrumento representan los diferentes elementos de la propiedad que se pretende medir.

Siguiendo la propuesta anterior, se realizó la valoración de contenido por medio de juicio de experticia, a quienes se les dio instrucciones específicas sobre el propósito general de la prueba, la conceptualización del universo de contenido, el plan de operacionalización. Se utilizó una tabla de cotejo que consideró: a) número de ítem, b) relevancia o congruencia, c) claridad en la redacción, d) imparcialidad o sesgo en la formulación, e) dimensión o dominio Volumen 11, Número 1, Año 2011, ISSN 1409-4703 
cognoscitivo, y f) nivel de dificultad. A partir de los criterios b), c) y d), los ítemes que resultaron con un $100 \%$ de coincidencia en su evaluación se mantuvieron, los que mostraron coincidencia parcial de las personas expertas se replantearon y los que tenían un $100 \%$ de condición desfavorable se eliminaron. A partir de los criterios e) y f) se verificó el equilibrio entre los diferentes niveles de dificultad y dominios cognoscitivos. Después de la validación, el instrumento definitivo estaba conformado por veintisiete ítemes, trece del área de conocimientos fácticos (saber), nueve de saber hacer, procedimental y cinco de actitudes, valores y disposición (ser y convivir).

El análisis de confiabilidad del instrumento se realizó mediante la prueba estadística Alpha de Crombach, la cual arrojó un coeficiente de correlación entre los factores que se midieron de 0,809. Ruiz (1998) considera que la confiabilidad se enfoca en el grado de homogeneidad de los ítemes del instrumento en relación con la característica que pretende medir, la cual basada en la teoría del error de medición implica que a mayor error implícito menor confiabilidad y a menor error mayor confiabilidad.

Tomando en cuenta que la escala de interpretación de la magnitud del coeficiente de confiabilidad difiere de cada situación particular, no obstante, se encuentra semejanza en la interpretación de resultados en diversos estudios de rendimiento académico y las escalas propuestas, de las cuales se cita a Ruiz (1998), quien considera que el rango entre 0,61 y 0,80 tiene una magnitud alta y que para ser aceptable debe concentrarse en el límite superior 0,80; sin embargo, aclara, citando a (Thorndike 1989 y Magnusson, 1986), que la literatura reporta coeficientes resultados en pruebas de rendimiento académico que pueden variar entre 0,61 y 0,80 .

Para la revisión de la prueba, una vez aplicada, se aplicó el criterio de fiabilidad interjueces aplicado por las dos investigadoras. Para ello, se estableció una escala cuantitativa que facilitara los posteriores análisis psicométricos en el programa SSPSS. A partir de los criterios cualitativos establecidos en las preguntas de respuesta abierta, las investigadoras le asignaron las puntuaciones $1=$ correcta $0=$ incorrecta, de esta manera se establece el criterio de fiabilidad interjueces para la corrección, la comparación de ambas revisiones no mostró diferencia significativa.

El procedimiento fue aplicar tratamiento al grupo experimental, es decir, se aplicó la propuesta de estrategias metodológicas para la producción de documentos en el modelo por competencias. Los otros dos grupos se asumieron como grupos control, los cuales recibieron el mismo curso con un programa de trabajo con una orientación de la enseñanza tradicional. 
Para el análisis de los resultados, se analizaron los datos con el paquete estadístico SPSS, versión SPSS Statistics 17,0. Se usaron estadísticas como la media y la relación del nivel de rendimiento con las variables trabajo, dual y carrera. La escala de valoración del rendimiento considerada con una nota según respuestas correctas con reescalamiento de índice de 0-100, a partir de las respuestas correctas-incorrectas, mínima y máxima, intragrupo. De esta escala se definieron tres niveles de rendimiento 0-33 = inferior, 34-66=intermedio y 67$100=$ superior .

\subsection{Técnicas cualitativas}

Para complementar los resultados de los test aplicados se exploraron algunos factores del proceso de enseñanza y de aprendizaje aplicado en cada grupo, mediante técnicas cualitativas: cuestionario y grupo focal, con preguntas abiertas y que se categorizaron en las dimensiones: estrategias de aprendizaje aplicadas, estrategias de aprendizaje que más favorecen su aprendizaje, coordinación interdisciplinaria, papel de la persona estudiante (empeño, dedicación y actitudes que asumió durante el curso), características de la persona docente del curso, experiencia laboral, participación en el Proyecto de Formación Dual y la carrera.

\section{RESULTADOS, ANÁLISIS Y DISCUSIÓN}

Se determinó el rendimiento en la producción de documentos a los grupos participantes en el cuasiexperimento, se detallan los resultados del pretest y del postest aplicados al grupo experimental y a los dos grupos control.

Como se observa en el Gráfico No. 1, en el grupo experimental, después de aplicada la propuesta de enseñanza por competencias, se observa una mejora de 13,3 puntos en el promedio de rendimiento, entre el pretest y el postest. Los grupos control, quienes, según el

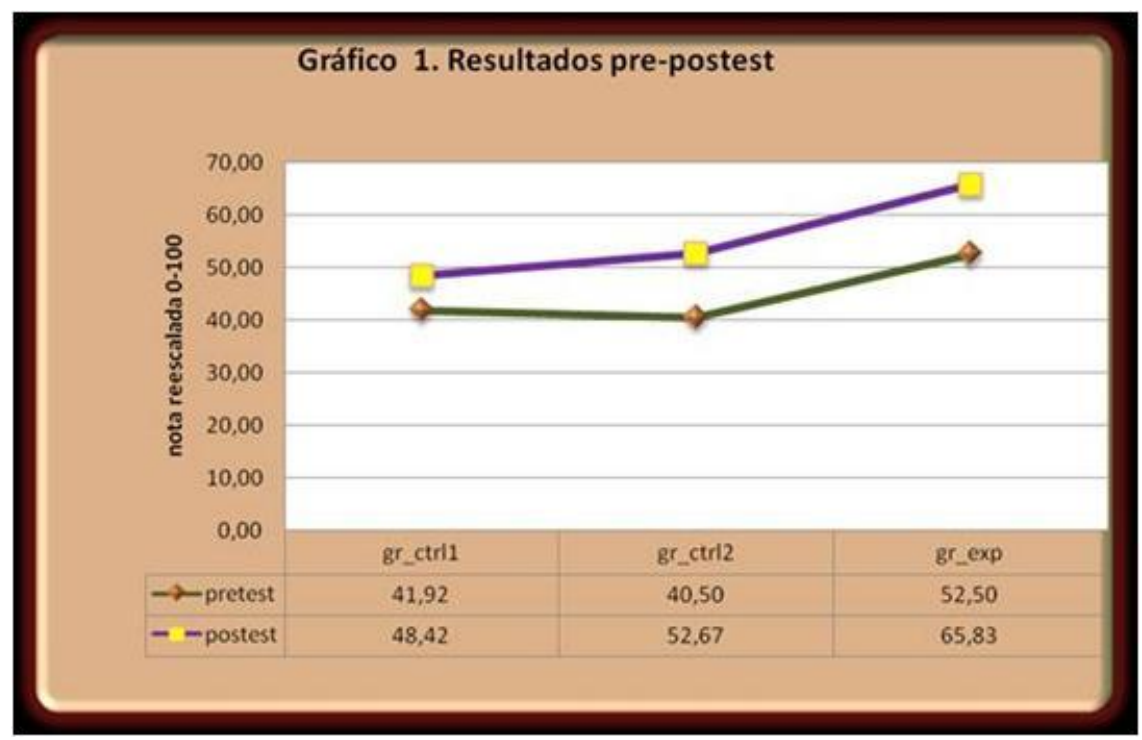


programa del curso utilizaron la metodología tradicional, muestran también una mejora para el grupo 1 del 6,5 y para el grupo 2 de 12,17 entre ambas pruebas.

En la Tabla No. 3 se presenta la comparación de los grupos, según los promedios intragrupo, por área cognoscitiva, según resultados del pretest y postest.

\begin{tabular}{|c|c|c|c|c|c|c|}
\hline \multicolumn{7}{|c|}{$\begin{array}{c}\text { Tabla No. 3. Comparación del Rendimiento } \\
\text { (por áreas o dimensiones cognoscitivas, por grupo, por test) }\end{array}$} \\
\hline & \multicolumn{2}{|c|}{ grup ctrl1 } & \multicolumn{2}{|c|}{ grup ctrl2 } & \multicolumn{2}{|c|}{ grup exp } \\
\hline- & $\underline{\text { pret }}$ & $\underline{\text { post }}$ & $\underline{\text { pret }}$ & $\underline{\text { post }}$ & $\underline{\text { pret }}$ & $\underline{\text { Postes }}$ \\
\hline Dimensión & \multicolumn{6}{|c|}{ Media } \\
\hline saber (conocimientos fácticos) & 0,91 & 6,91 & 1,09 & 5,82 & 0,82 & 10,27 \\
\hline hacer (saber hacer, precedimental) & 5,00 & 6,73 & 3,00 & 6,36 & 3,64 & 8,55 \\
\hline $\begin{array}{l}\text { ser y convivir (actitudes, valores, } \\
\text { disposición) }\end{array}$ & 7,2 & 9,2 & 7,2 & 10,2 & 6,2 & 11,2 \\
\hline
\end{tabular}

En esa tabulación es importante resaltar los resultados del pretest por dimensiones cognoscitivas, repunta el grupo experimental, lo que refleja un proceso de enseñanza desarrollado para fortalecer esas diferentes áreas de conocimiento. Cabe observar que los tres grupos tienen altos índices en las áreas de ser y convivir.

El nivel de rendimiento intragrupos, tanto del pretest como del postest, se muestra en el Gráfico No. 2. Como era de esperarse, antes del tratamiento, los tres grupos mostraron mayor

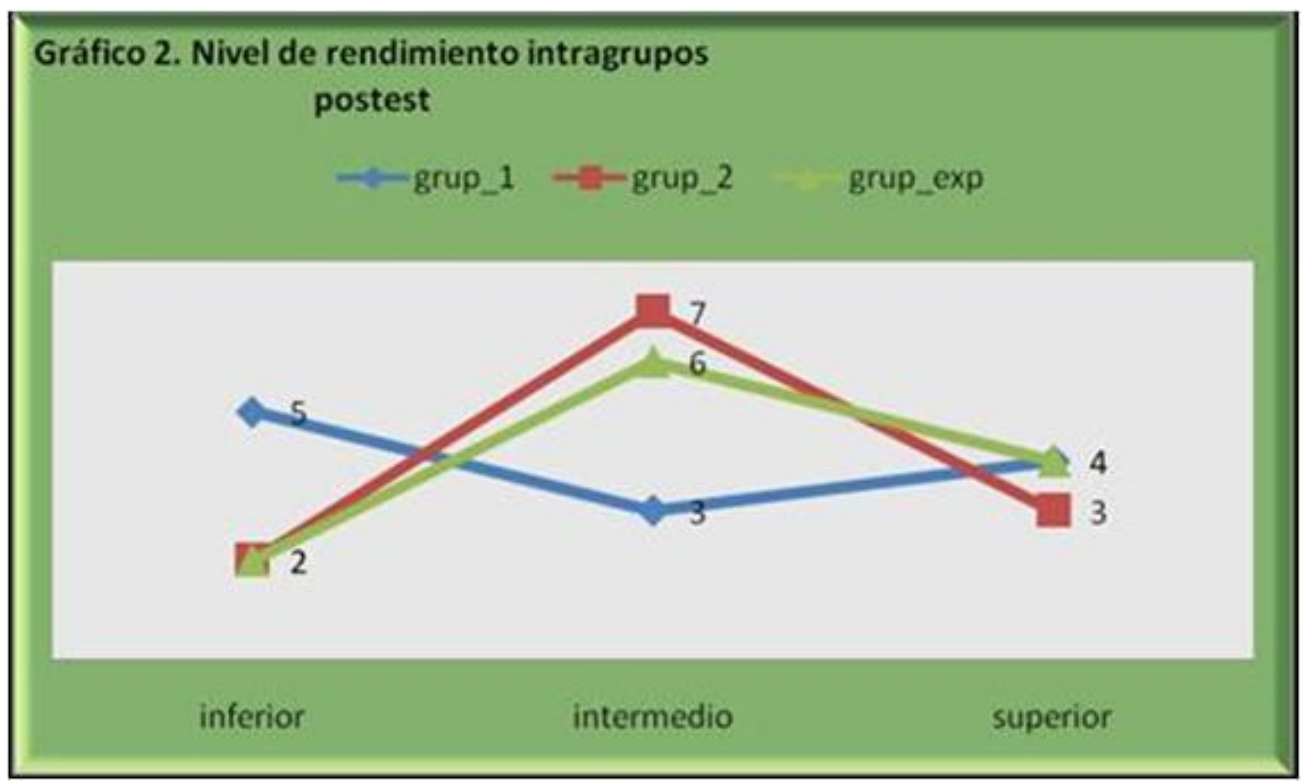


incidencia en el nivel inferior y en el postest se aprecia una mejora significativa para los tres grupos. Es importante anotar que el grupo control 2 supera al grupo experimental en cuanto a personas estudiantes con nivel intermedio, pero ambos muestran una mejora en ese nivel.

El nivel de rendimiento se asocia tanto a conductas propias de la persona estudiante, como elementos del proceso de enseñanza y aprendizaje aplicado. Estas se detallan, según niveles de rendimiento definidos como inferior, intermedio y superior, la mayor aplicación en cada grupo.

Al relacionar los elementos del proceso de enseñanza y de aprendizaje con los resultados del cuasiexperimento, a partir de la opinión de la población participante, se identifica la incidencia de esos aspectos en el aprovechamiento logrado en el curso.

En el grupo experimental, las estrategias más utilizadas están relacionadas con técnicas experienciales. La toma de actas en un órgano colegiado y el trabajo colaborativo fueron de las actividades que más les gustaron a la población participante, quienes indican haber sentido satisfacción porque fue una actividad interesante, agradable y bonita, pero a la vez sintieron presión, nervios y cansancio.

Las estrategias de aprendizaje llevadas a cabo en el curso, en cada uno de los grupos, según indicación de los estudiantes y de las estudiantes, se muestran las siguientes, en orden de mayor frecuencia (ver Tabla No. 4).

\begin{tabular}{|l|l|}
\hline \multicolumn{2}{|c|}{$\begin{array}{c}\text { Tabla No. } 4 . \\
\text { (En orden de mayor frecuencia) }\end{array}$} \\
\hline \multicolumn{1}{|c|}{ grupo experimental grupos control } \\
\hline $\begin{array}{l}\text { Trabajo colaborativo (en parejas o } \\
\text { grupos) }\end{array}$ & Trabajo individual en el computador \\
\hline Toma de actas en órgano colegiado & Práctica dirigida \\
\hline Simulación de actividades & Trabajo individual con libro de texto \\
\hline Visita a salas de reuniones & Elaboración de actas según formato del libro \\
\hline Elaboración de mapas conceptuales & Análisis de texto (lecturas) \\
\hline Entrevista a especialistas & Toma de actas en órgano colegiado \\
\hline Cuadros comparativos & Exposición de la profesora \\
\hline Trabajo individual en el computador & Elaboración de mapas conceptuales \\
\hline $\begin{array}{l}\text { Elaboración de informes (trabajo } \\
\text { interdisciplinario) }\end{array}$ & \\
\hline Talleres & \\
\hline Exposición de la profesora & \\
\hline Fuente: Elaboración de las autoras &
\end{tabular}


El logro de coordinación interdisciplinaria entre el curso en estudio con otros cursos de la carrera, del mismo nivel, se muestra muy limitada y la única actividad que se evidencia en el grupo experimental es la realización de un informe programado por la profesora del curso de Comunicación y Redacción en coordinación con el curso Técnicas Mecanográficas. En esta actividad, las personas estudiantes abordaron un problema de la práctica profesional desde dos perspectivas, el formato y la redacción, actividad que para el estudiantado resultó satisfactoria porque cumplieron una sola tarea para dos cursos y lograron producir un documento de manera completa.

El papel de la persona estudiante, en cuanto al empeño en aprender y desarrollar habilidades durante el curso, lo valoran, en su mayoría, en los tres grupos, entre un $60 \%$ y un $70 \%$. Esta situación se relaciona con los resultados de los tests, entre más empeño en aprender y desarrollar habilidades mayor nivel de rendimiento, de esta manera se confirma su interés en el proceso de enseñanza y de aprendizaje y su mejora en el rendimiento.

En el grupo experimental, a pesar de que prevalecieron actitudes positivas, intereses y motivaciones por aprender cada día, destacaron la presión de trabajo, temores para afrontar situaciones de aprendizaje nuevas, el poco tiempo para desarrollar tareas en clase, la frustración por falta de equipo de cómputo en la casa, el cansancio y la ansiedad ante la diversidad de actividades y obligaciones que exigió el curso. Al solicitarles que anotaran tres actitudes que asumieron durante el curso, mencionan las siguientes:

\begin{tabular}{|l|l|l|}
\hline \multicolumn{3}{|c|}{ Actitudes que las Personas Estudiantes Asumieron Durante el Curso } \\
\hline \multicolumn{1}{|c|}{ Grup_1 } & \multicolumn{1}{|c|}{ Grup 2 } & \multicolumn{1}{c|}{ Grup_exp } \\
\hline Interés, concentración, & Ganas de aprender & La responsabilidad, análisis \\
positivismo (sí lo puedo & (superarme), actitud & de actas e informes, realizar \\
lograr) & positiva & activides fuera del aula \\
responsabilidad, respeto, & perseverancia, \\
tolerancia, deseos de & responsabilidad, orden de actas) \\
aprender & precisión & constancia, deseos de \\
las ganas de aprender, & práctica, observación & trabajo colaborativo, \\
esfuerzo & comunicación, & experiencia laboral, \\
Poner atención y evacuar & pensamiento, & disposición \\
dudas, tomar apuntes y leer & destrezas & tolerancia, práctica, \\
los libros, hacer prácticas, & tomar o ser partícipe & experiencia \\
la disposición, el empeño y & en un órgano & positivismo \\
leer mucho & colegiado & individuales, tareas \\
estar atento, entender, saber & disciplina &
\end{tabular}




\begin{tabular}{|l|l|l|}
\hline $\begin{array}{l}\text { analizar } \\
\text { la buena disposición de la } \\
\text { profe, el orden del grupo y lo } \\
\text { diversa que es la clase } \\
\text { claridad de explicación, } \\
\text { interés } \\
\text { responsabilidad, iniciativa, } \\
\text { eficiencia } \\
\begin{array}{l}\text { concentración, respeto, } \\
\text { interés, organización, orden } \\
\text { ser atento. }\end{array}\end{array}$ & $\begin{array}{l}\text { la seguridad de sí } \\
\text { misma, la } \\
\text { las prácticas, las } \\
\text { tareas } \\
\text { trabajo grupal, } \\
\text { coordinación. }\end{array}$ & $\begin{array}{l}\text { talleres, trabajos en grupos, } \\
\text { supervisión profesora, } \\
\text { práctica personal } \\
\text { responsabilidad, } \\
\text { puntualidad, poner atención } \\
\text { la paciencia, las ganas de } \\
\text { aprender, la superación } \\
\text { personal } \\
\text { perseverancia, comprensible } \\
\text { e interés por aprender. }\end{array}$ \\
\hline \multicolumn{2}{|l|}{\begin{tabular}{l} 
Fuente: Elaboración de las autoras \\
\hline
\end{tabular}} \\
\hline
\end{tabular}

Sobre las cualidades o características de la profesora de cada grupo, subrayan las cualidades humanas, seguido de la didáctica y pedagogía aplicada y en tercer lugar mencionan el conocimiento técnico.

Las personas participantes, en los tres grupos, consideran a cada una de las profesoras como una profesional de gran experiencia, que les daba seguridad de lo que ella explicaba. De la profesora del grupo experimental enfatizan que tenía conocimiento y disposición para enseñar, de realizar clases prácticas, de realizar charlas y conferencias con expertos para brindarles su conocimiento, además del buen trato y la motivación ejercida.

Se presentan las características más mencionadas y los aspectos que valoran en la persona docente:

- Actualización en la materia.

- Si me impresionan se aprende más.

- Creatividad y más metodología.

- Crear conocimiento entre todos.

- Innovación.

- Que no sea estricto ni pasivo (intermedio).

- Abierto a escuchar sugerencias.

- Flexible a necesidades de los estudiantes y de las estudiantes.

- Dinamismo.

- Comprensivo.

- Equitativo.

- Gran ser humano.

- Posea valores. 
- Entorno de respeto.

- Presentación personal.

- Responda inquietudes.

- Buena relación humana.

- Que sea paciente.

- Aplicación correcta de contenidos.

- Respetuoso.

Al relacionar la experiencia laboral con el nivel de rendimiento, en los grupos experimental y grupo control 1 , se presenta que las personas que trabajan, en su mayoría, se posicionan en los niveles de rendimiento intermedio y superior; sin embargo, en el grupo control 2, ninguno trabaja y en general muestran un mejor rendimiento que el grupo control 1. Por eso, y en este caso, no puede deducirse que la experiencia laboral sea un factor que incide en el rendimiento y que necesite estudio más profundo.

La Formación Dual ${ }^{4}$ es un aspecto que podría determinar el rendimiento de manera creciente, las personas estudiantes que han participado en este proyecto poseen experiencia en labores relacionadas con la producción de documentos y se indica el logro de niveles de rendimiento entre intermedio y superior.

El curso Técnicas Mecanográficas por Computadora III es un curso base para las dos carreras: Licenciatura en Administración de Oficinas y Licenciatura en Educación Comercial, y las cifras revelan que para la primera un $44 \%$, el nivel de rendimiento se encuentra en el nivel superior y un $36 \%$ en nivel intermedio, mientras que para la segunda un $20 \%$ se concentra en nivel superior y un $40 \%$ en intermedio. Las personas estudiantes que cursan las dos carreras presentan índices de rendimiento en los niveles superior e intermedio de un 33\%.

También, es importante agregar que las personas estudiantes del grupo experimental indicaron que fue un curso muy provechoso; no obstante, les generó un esfuerzo extra, y por ello anotaron los siguientes elementos:

- Fue un curso muy cargado.

- Un ambiente ameno.

4 Formación en alternancia Universidad-Empresa que la Escuela de Secretariado Profesional ofrece a los estudiantes y las estudiantes del segundo año de la carrera de Administración de Oficinas, durante un año lectivo para un total de 600 horas anuales. 
- Son temas importantes para la carrera.

- Muchas tareas (tedioso).

- Mucha presión.

- $\quad$ Frustración por falta de equipo.

- Cansancio en las últimas lecciones.

- Buen aprendizaje.

- Frustración por falta de equipo en la casa.

- Les gustó trabajar con el aula virtual.

- Se quejaron de falta de tiempo para el desarrollo de los temas, ya que se vieron de forma muy amplia.

- La atención de la profesora fue una fortaleza.

- El método de aprendizaje fue muy interesante, ya que es muy innovador aprender por competencias sabiendo que lo que trabajamos en clase corresponde a la realidad del desempeño de la profesión en alguna institución o empresa, pública o privada.

- Puede ser que la mayoría de trabajos fueran extensos, por lo que no daba tiempo de terminarlos en clase, eso implicó buscar medios fuera del aula (computadora y la Internet) para concluirlos.

- Debido a que estaba llevando muchos cursos, no pude dedicarle el tiempo necesario; sin embargo, sí comprendí los temas y entendí como escribir los documentos.

- El tiempo del curso no nos alcanzó muchas veces para finalizar los trabajos de clase, por lo que quedaban como tareas.

- Poco tiempo, pues faltó tiempo en las lecciones para desarrollar más los temas.

- El compañerismo que permitió el trabajo en equipo y se puede trabajar con cualquier compañero, aportar ideas, compartir conocimientos y experiencias, por ejemplo, el examen final (en parejas) me pareció una gran idea que aportó mucho para el aprendizaje.

- Permite vivenciar lo estudiado en clase en ambientes reales.

- Estuvo dinámica y diferente. 


\section{CONCLUSIONES}

El proceso de enseñanza y de aprendizaje basado en el modelo por competencias podría mejorar el rendimiento académico de las personas estudiantes y acercar el conocer a la actividad experiencial y a la práctica en contextos más parecidos al futuro campo laboral.

Aunque desde las instituciones educativas se quiera integrar la teoría con la práctica, esto es posible solamente en cierta medida siguiendo el modelo tradicional. En cambio, la formación por competencias introduce variaciones a la actividad educativa comercial a partir de experiencias en otros contextos o por medio de actividades más relacionadas con la realidad, como es el caso de las pasantías para la toma de actas en una sesión de órgano colegiado o una entrevista con un especialista. Por lo tanto, el potencial desde las aulas se visualiza en el cambio de la metodología y de las actividades de aprendizaje que involucran la interdisciplinariedad, el diálogo entre la persona docente, la persona estudiante y las personas profesionales en servicio, así como la indagación del campo de la especialidad.

Es importante tomar en cuenta que tanto en los grupos control como en el grupo experimental se aplicaron técnicas participativas, resolución de casos, actividades didácticoproductivas, las cuales están asociadas al rendimiento académico. En el grupo experimental se evidencia el énfasis en actividades experienciales que permiten abordar los problemas de la práctica profesional propuestos en el programa del curso y que se abordaron en el desarrollo de las competencias específicas, genéricas en la integración del conocimiento; esto se desarrolló con el aporte e intervención de personas especialistas en los talleres, con la cooperación de personas expertas en las instituciones en el caso de las pasantías, en la indagación sobre la temática; también, fue relevante el trabajo en equipo de personas estudiantes y el abordaje de tareas para producir los documentos desde diversos ámbitos: aspectos de formato, de contenido, de redacción, legales, administrativos, orientación de aprendizaje que fundamentan un proceso interdisciplinario para el desarrollo de la competencia en producción documental.

Esta fue la respuesta a un programa de estudios que sólo ubica en el mismo nivel los cursos que abordan, uno la redacción y la gramática y otro el formato y la herramienta tecnológica, por lo tanto, la persona docente del grupo experimental debió integrar con especialistas en la materia, con fuentes bibliográficas y su propia experticia, los conocimientos relativos a los procedimientos administrativos y legales.

Otra situación que es importante resaltar y que incumbe las competencias con la integración de saberes es la relación positiva encontrada entre el nivel de rendimiento Volumen 11, Número 1, Año 2011, ISSN 1409-4703 
intermedio y superior en estudiantes que participaron en el Proyecto de Formación Dual en puestos de oficina. Esta variable, de la cual la Unidad Académica en estudio tiene vasta experiencia, es un potencial para considerar en nuevas propuestas de enseñanza por competencias.

La metodología empleada en la propuesta por competencias, cuyo énfasis es participativa, en la que prevalece el aprendizaje cooperativo, así como la orientación de la persona docente en actividades que generen el análisis, diálogo, indagación, la participación en actividades lo más cercanas a la realidad práctica del futuro ejercicio profesional, además del interés y motivación de las personas participantes. Este aspecto es importante considerarlo, por ejemplo, en el grupo experimental, las personas estudiantes indicaron que prevalecieron actitudes positivas, intereses y motivaciones por aprender cada día, pero destacaron la presión de trabajo, temores para afrontar situaciones de aprendizaje nuevas, el poco tiempo para desarrollar tareas en clase, la frustración por falta de equipo en la casa, el cansancio y la ansiedad ante la diversidad de actividades y obligaciones que exigió el curso, es decir, la mejora en el rendimiento mostrado significó un gran esfuerzo y dedicación de parte de ellos y respondió también al interés e identificación de la persona docente.

En ese sentido, desde la docencia, involucrar a la persona estudiante en estrategias de aprendizaje que le exigen la investigación, la realización de tareas propias de la realidad productiva, promover enlaces de cooperación con el sector empresarial para que tenga la oportunidad de aplicar la teoría a la resolución de problemas reales, favorecen el aprendizaje y desarrollo de las competencias requeridas por un perfil profesional futuro, aspectos que la Universidad Nacional definió en el modelo pedagógico.

Este trabajo ha permitido evidenciar que la transmisión de contenidos en clase, por medio de un texto o de la exposición reiterada por parte de la persona docente, es una forma tradicional de enseñar y no de propiciar espacios para construir y reconstruir con el fin de integrar saberes. De ahí que la necesidad por establecer nuevas formas para el proceso de enseñanza y aprendizaje, surgida a raíz del constante cambio de los medios productivos, las condiciones culturales, formas de pensar y de actuar necesitan un análisis profundo para la búsqueda de soluciones a los problemas humanos.

La persona estudiante, en la actualidad, no es receptora, es una gestora o constructora del conocimiento en una realidad suya, con sus retos. También, la persona docente ha pasado a ser aprendiz de nuevas realidades. En esta circunstancia, la indagación para contar con 
información desde diversas fuentes, teóricas, experienciales y de visión futura es la manera que permite ofrecer aportes para que la sociedad logre enfrentar las situaciones difíciles.

A partir de la aplicación de la propuesta de enseñanza por competencias se evidencia la integración del saber conocer, del saber hacer, del ser y del convivir, pero hace falta un estudio más profundo sobre la situación curricular en cuanto a tiempo, condiciones materiales y humanas de los cursos para ponerlos en ejecución en este enfoque pedagógico.

A partir de lo anterior, se recomienda tomar en cuenta para el abordaje de un proceso educativo por competencias, los siguientes aspectos:

1. Que la unidad académica aborde la temática de enseñanza por competencias con una visión integradora basada en los pilares fundamentales de la Educación que propone la UNESCO: Saber, saber hacer, ser y convivir, de manera que la búsqueda de las soluciones de los problemas de la práctica profesional potencie el aprendizaje, con miras a una formación profesional con alta capacidad productiva y comprometido con los valores humanos para la convivencia y desarrollo de sus potencialidades, sin estos elementos no hay enseñanza basada en competencias Conviene, para ello, la gestión desde las autoridades de la unidad académica la promoción de este enfoque pedagógico.

2. Al planificar un curso o una carrera en el modelo por competencias, estudiar las posibilidad teórica y metodológica que involucra un propósito general o competencia global, la cual pretende la construcción del conocimiento a partir de la búsqueda de solución a los problemas de la práctica profesional, de allí se derivan las competencias específicas, los elementos de competencia y sus ejes temáticos, las competencias genéricas como ejes transversales que fortalecen el currículo en cuanto actitudes y valores. Además, considerar los indicadores de logro o estrategia evaluativa, tanto de las competencias específicas como de las genéricas, aplicables al inicio, en el proceso y al final, así como varias fuentes de consulta actualizadas y la relación obligada del contexto profesional. Para la definición del propósito general o competencia global de un curso es relevante un diagnóstico curricular orientado a la determinación del perfil profesional que se desea formar.

3. En el abordaje de un proceso de enseñanza y de aprendizaje en el modelo por competencias resaltar: la metodología de enseñanza y de aprendizaje dinámica e interactiva, dialógica; la persona docente es facilitadora; la persona estudiante activa y responsable de su propio aprendizaje, analítica, indagadora, propositiva; aplicación de 
situaciones de aprendizaje que confronten las propuestas teóricas con el estudio de la realidad, por medio de aprendizaje experiencial en el aula y en entornos lo más relacionados con la futura práctica profesional; la interdisciplinariedad como eje fundamental en la integración de saberes; y la evaluación como medio para aprender, porque para el logro de aprendizajes significativos es necesaria la interacción de los conocimientos previos con los conocimientos nuevos y de su adaptación al contexto y que además va a ser funcional en determinado momento de la vida del individuo.

4. La coordinación interdisciplinaria con los demás cursos de la carrera. Los que involucran producción de documentos, cuyo énfasis está en las técnicas de digitación (formato) y de contenido, se aborden desde una perspectiva más completa o integrando elementos de formato, legales, gramaticales, procedimientos administrativos, así como actitudes y valores. Una forma oportuna de esa integración interdisciplinaria y que favorece el aprendizaje es la resolución de casos, tareas o proyectos coordinados por los diferentes cursos, de tal manera que haya un desarrollo completo de la competencia específica. Para ello, es necesario revisar la malla curricular y en los cursos indicados aplicar la horizontalidad curricular requerida.

5. Fortalecer la práctica del Proyecto de Formación Dual en el plan de estudios de las dos carreras que ofrece la Escuela de Secretariado Profesional, pues es una estrategia de aprendizaje didáctica-productiva y enriquecería un proceso de enseñanza por competencias.

6. Fortalecer la herramienta del aula virtual como una posibilidad para incorporar un componente comunicativo permanente entre las personas participantes en un proceso pedagógico, facilitar el manejo y organización de recursos didácticos, además, las sesiones virtuales posibilitan el aprovechamiento del tiempo para indagar la realidad productiva.

7. Generar más actividades de investigación que profundicen sobre la temática, específicamente elementos sobre la situación curricular: tiempo, condiciones materiales y humanas de los cursos para ponerlos en ejecución en el modelo por competencias, considerando que este modelo pedagógico se potencia como una oportunidad para mejorar la calidad educativa. 


\section{Referencias}

Alonso, Catalina, Gallego, Domingo y Honey, Peter. (1994). Los Estilos de Aprendizaje, Procedimientos de Diagnóstico y Mejora. Bilbao, España: Ediciones Mensajero.

Araya, Isabel. (2008). La Formación Dual y su Fundamentación Curricular. Educación: Revista de la Universidad de Costa Rica, 32(1),45-61. San José, Costa Rica.

Badilla, Leda. (s.f.). Documentos sobre algunos aportes al concepto de competencias desde la perspectiva de América Latina. Recuperado el 12 de noviembre de 2008, de http://www.cumex.org.mx/archivos/ACERVO/Tuning.pdf

Beneitone, Pablo, Esquetini, César, González, Julia, Maletá, Maida Marty, Siufi, Gabriela y Wagenaar, Robert. (2007). Reflexiones y perspectivas de la Educación Superior en América Latina, Informe Final, Proyecto Tuning América Latina 2004-2007. Disponible en http://tuning.unideusto.org/tuningeu/.

Cortés, Angela. (1984). Métodos para la Enseñanza de la Mecanografía. Colombia: McGrawHill Latinoamericana.

Costa Rica. (1989, 21 de julio). Código Procesal Civil. Ley No. 7130. Publicado en La Gaceta No. 208 de 3 de noviembre de 1989. San José, C.R.: Asamblea Legislativa.

Costa Rica. (1990, 24 de octubre). Ley del Sistema Nacional de Archivos No. 7202 y Reglamento. Cuadernillos del Archivo Nacional. No. 4. Dirección General del Archivo Nacional Ministerio de Cultura, Juventud y Deportes. San José, Costa Rica: Asamblea Legislativa.

Costa Rica, Consejo Nacional de Rectores. (2005). Plan Nacional de Educación Superior Universitaria Estatal 2006-2010. San José, Costa Rica: CONARE-OPES (Oficina de Planificación de la Educación Superior)

Costa Rica, Junta Administrativa del Archivo Nacional. (2007). Directriz 29-2007. La Gaceta 61 - Viernes 28 de marzo del 2008 (Fe de Erratas: Gaceta 83-2008).

Delors, Jaques, Mufti, In`am Al, Amagi, Isao, Carneiro, Roberto, Chung, Fay, Geremek, Bronislaw, Gorham, William, Kornhauser, Neksandra, Manley, Michael, Padrón, Marisela, Savané, Marie-Angélique. Singh, Karan. Stevenhager, Rodolfo, Won Suhr, Myong y Nanzhao, Zhou. (1996). La Educación Encierra un Tesoro. Informe a la UNESCO de la Comisión Internacional sobre la Educación para el Siglo XXI. París: Ediciones UNESCO.

Demagistri, María Silvina. (2009). Habilidades cognitivas y rendimiento académico en alumnos de una escuela secundaria. Recuperado el enero de 2009, de Base de datos Fuente Académica, EBSCO host.

Dewey, John. (1960). Experiencia y Educación. Buenos Aires, Argentina: Ed. Losada. 
DRAE (2001). Diccionario de la lengua española (22a ${ }^{a}$. Ed.). Recuperado el 20 de noviembre de 2010, de http://www.rae.es/drael/.

García, Ma. Visitación, Alvarado, Jesús Ma. y Jiménez, Amelia. (2000). La predicción del rendimiento académico: regresión lineal versus regresión logística. Psicothema, 12, Supl. (2), 248-252, Universidad Complutense de Madrid. Recuperado el 23 de marzo de 2009, de http://www.psicothema.com/pdf/558.pdf

Gimeno, José, Pérez, Angel, Martínez, J. Bautista, Torres, Jurjo, Angulo, Félix y Álvarez, Juan Manuel. (2009). Educar en competencias, ¿qué hay de nuevo? Madrid, España: Ediciones Morata, S.L.

Hernández, Eugenia, Hernández, Digna y Segura, Lilliam. (2005). Gestión Documental y Administración Electrónica de Documentos. San José, Costa Rica: Litografía e Imprenta Lil, S.A.

Kemmis, Stephen. (1998). El curriculum: más allá de la teoría de la reproducción. Traducción de Curriculum tehorising: Beyond Reproduction Theory. 3ra. Ed. Madrid, España: Ediciones Morata, S.L.

Kerlinger, Frederick y Lee, Howard B. (2002). Investigación del Comportamiento (4ª . Ed.). México: McGraw Hill.

Molero, Lopez, Barajas, David. (2007). Rendimiento académico y opinión sobre la docencia del alumnado participante en experiencias piloto de implantación del Espacio Europeo de Educación Superior. RELIEVE, 13 (2). Recuperado el 15 de abril de 2008, de http://www.uv.es/RELIEVE/v13n2/RELIEVEv13n2 2.htm.

Monereo, Carles (coord.), Castelló, Monserrat, Clariana, Mercé, Palma, Monserrat, Pérez. María L. (1999). Estrategias de enseñanza y aprendizaje. Formación del profesorado y aplicación a la escuela. Barcelona: Graó Editorial.

Montero, Eiliana, Villalobos, Jeannette y Valverde, Astrid. (2007). Factores institucionales, pedagógicos, psicosociales y sociodemográficos asociados al rendimiento académico en la Universidad de Costa Rica: Un análisis multinivel. RELIEVE, 13 (2), 215-234. Recuperado el 15 de abril de 2010, de http/www.uv.es/RELIEVE/v13n2/RELIEVEv13n2 5.htm

Perrenoud, Philippe. (2007). Diez nuevas competencias para enseñar. Barcelona (5a. ed.). España: Graó.

Pozo, Juan Ignacio. (1990). Estrategias de aprendizaje. En C. Coll, J. Palacios y A. Marchesi (Comps.), Desarrollo psicológico y educación II. Psicología de la Educación (pp. 199221). Madrid: Alianza.

Ruiz, Carlos. (1998). Instrumentos de Investigación Educativa. CIDEG. Barquisimeto. Recuperado el 15 de junio de 2010, de http://www.carlosruizbolivar.com/articulos/archivos/Curs0\%20C\|\%20\%20UCLA\%20Art.\% 20Confiabilidad.pdf. 
Tobón, Segio. (2005). Formación Basada en Competencias (2a. Ed.). Bogotá, Colombia. Ecoe Ediciones.

Tünnermann, Carlos. (1997). La educación para el siglo XXI. Caracas, Venezuela: UNESCO.

Universidad Nacional de Costa Rica. (s.f.). Modelo Pedagógico. Heredia, Costa Rica: UNA.

Universidad Nacional de Costa Rica. (2004). Plan Global Institucional 2004-2011. Asamblea de Representantes. Heredia: Departamento de Publicaciones de la Universidad Nacional.

Villa, Aurelio y Poblete, Manuel. (2004). Practicum y evaluación de competencias. Revista de currículo y formación del profesorado, 8, 1-19. Recuperado el 12 de noviembre de 2007, de hppt/www.redalyc.uaemex.mx/pdf/206/20611455007.pdf

Villarini, Angel. (1996). El Currículo Orientado al Desarrollo Humano Integral. Puerto Rico: Organización para el Fomento del Desarrollo del Pensamiento.

Zapata, Luis Felipe, De los Reyes, Carlos, Lewis, Soraya. y Barceló, Ernesto. (2009). Memoria de Trabajo y Rendimiento Académico en Estudiantes de Primer Semestre de una Universidad de la Ciudad de Barranquilla. Revista Psicología desde el Caribe, 23, 6682. Recuperado 22 de agusto de 2010. De Base de Datos Fuente Académica, EBSCOhost. 